16. Meyer, M., and Wagner, E. 2006. Recent developments in the application of plasmid DNA-based vectors and small interfering RNA therapeutics for cancer. Hum. Gene Ther. 17:1062-1076.

17. Xia, X., Zhou, H., Huang, Y., and Xu, Z. 2006. Allele-specific RNAi selectively silences mutant SOD1 and achieves significant therapeutic benefit in vivo. Neurobiol. Dis. 23:578-586.

18. Yang, T.P., Stout, J.T., Patel, P.I., Alsford, R.L., and Caskey, C.T. 1988. Spontaneous reversion of novel LeschNyhan mutation by HPRT gene rearrangement. Somat. Cell Mol. Genet. 14:293-303.

19. Jonkman, M.F. 1999. Revertant mosaicism in human genetic disorders. Am. J. Med. Genet, 85:361-364.
20. Bittar, M., and Happle, R. 2005. Revertant mosaicism and retrotransposons: another explanation of "natural gene therapy". Am. J. Med. Genet. A. 137:222.

21. Pizzuti, A., Pieretti, M., Fenwick, R.G., Gibba, R.A., and Caskey, C.T. 1992. A transposon-like element in the deletion-prone region of the dystrophin gene. Genomics. 13:594-600.

22. Thanh, L.T., Nguyen, T.M., Helliwell, T.R., and Morris, G.E. 1995. Characterization of revertant muscle fibers in Duchenne muscular dystrophy, using exon-specific monoclonal antibodies against dystrophin. Am. J. Hum. Genet. 56:725-731.

23. Jonkman, M.F., et al. 1997. Revertant mosaicism in epidermolysis bullosa caused by mitotic gene conversion. Cell. 88:543-551.

24. Bliksrud, Y.T., Brodtkorb, E., Andresen, P.A., van den Berg, I.E.T., and Kvittingen, E.A. 2005. Tyrosinaemia type I - de novo mutation in liver tissue suppressing an inborn splicing defect. J. Mol. Med. 83:406-410.

25. Wada, T., et al. 2005. Oligoclonal expansion of T lymphocytes with multiple second-site mutations leads to Omenn syndrome in a patient with RAG1deficient severe combined immunodeficiency. Blood. 106:2099-2101.

26. Mavilio, F., et al. 2007. Correction of junctional epidermolysis bullosa by transplantation of genetically modified epidermal stem cells. Nat. Med. 12:1397-1402.

\title{
Cellular and molecular basis of wound healing in diabetes
}

\author{
Harold Brem ${ }^{1}$ and Marjana Tomic-Canic ${ }^{2}$ \\ ${ }^{1}$ Wound Healing and Vascular Biology Laboratory, Division of Plastic Surgery, Columbia University College of Physicians and Surgeons, \\ New York, New York, USA. ${ }^{2}$ Tissue Repair Laboratory, Tissue Engineering, Regeneration, and Repair Program, and \\ Department of Dermatology, Hospital for Special Surgery, Weill Medical College of Cornell University, New York, New York, USA.
}

\begin{abstract}
Diabetic foot ulcers (DFUs), a leading cause of amputations, affect $15 \%$ of people with diabetes. A series of multiple mechanisms, including decreased cell and growth factor response, lead to diminished peripheral blood flow and decreased local angiogenesis, all of which can contribute to lack of healing in persons with DFUs. In this issue of the JCI, Gallagher and colleagues demonstrate that in diabetic mice, hyperoxia enhances the mobilization of circulating endothelial progenitor cells (EPCs) from the bone marrow to the peripheral circulation (see the related article beginning on page 1249). Local injection of the chemokine stromal cell-derived factor-1 $\alpha$ then recruits these EPCs to the cutaneous wound site, resulting in accelerated wound healing. Thus, Gallagher et al. have identified novel potential targets for therapeutic intervention in diabetic wound healing.
\end{abstract}

Diabetes affects approximately 170 million people worldwide, including 20.8 million in the USA (1), and by 2030 these numbers are projected to double (2). The foot ulcer is a leading cause of hospital admissions for people with diabetes in the developed world (3) and is a major morbidity associated with diabetes, often leading to pain, suffering, and a poor quality of life for patients. Diabetic foot ulcers (DFUs) are estimated to occur in $15 \%$ of all patients with diabetes (3) and precede $84 \%$ of all diabetes-related lowerleg amputations (4).

Nonstandard abbreviations used: DFU, diabetic foot ulcer; EPC, endothelial progenitor cell; HBO, hyperbaric oxygen therapy; SDF-1 $\alpha$, stromal cell-derived factor $-1 \alpha$.

Conflict of interest: The authors have declared that no conflict of interest exists.

Citation for this article: J. Clin. Invest. 117:1219-1222 (2007). doi:10.1172/JCI32169.
Despite the existence of protocols to standardize care, the physiological impairments that can result in a DFU complicate the healing process. Currently, the only FDAapproved growth factor and cell therapies for DFUs are not routinely used during treatment, preventing professionals from implementing evidence-based protocols (5).

\section{Molecular pathogenesis of diabetic wound healing}

The moment a person with diabetes suffers a break in the skin of their foot, they become at danger for amputation. Most commonly, patients have neuropathy, which could be causative. When coupled with an impaired ability to fight infection, these patients become largely unable to mount an adequate inflammatory response. Thus, the DFU that may look like a healing wound becomes a portal for infection that can lead to sepsis and require limb amputation.
Over 100 known physiologic factors contribute to wound healing deficiencies in individuals with diabetes (Figure 1). These include decreased or impaired growth factor production (6-8), angiogenic response $(8,9)$, macrophage function (10), collagen accumulation, epidermal barrier function, quantity of granulation tissue (8), keratinocyte and fibroblast migration and proliferation, number of epidermal nerves (11), bone healing, and balance between the accumulation of ECM components and their remodeling by MMPs (12). Wound healing occurs as a cellular response to injury and involves activation of keratinocytes, fibroblasts, endothelial cells, macrophages, and platelets. Many growth factors and cytokines released by these cell types are needed to coordinate and maintain healing.

Molecular analyses of biopsies from the epidermis of patients have identified pathogenic markers that correlate with delayed wound healing. These include overexpression of c-myc and nuclear localization of $\beta$-catenin (13). Coupled with a reduction in and abnormal localization of EGFR and activation of the glucocorticoid pathway, keratinocyte migration is inhibited $(13,14)$. At the nonhealing edge (callus) of DFUs, keratinocytes show an absence of migration, hyperproliferation, and incomplete differentiation $(13,14)$. Fibroblasts demonstrate a phenotypic change as well as decreased migration and proliferation. 


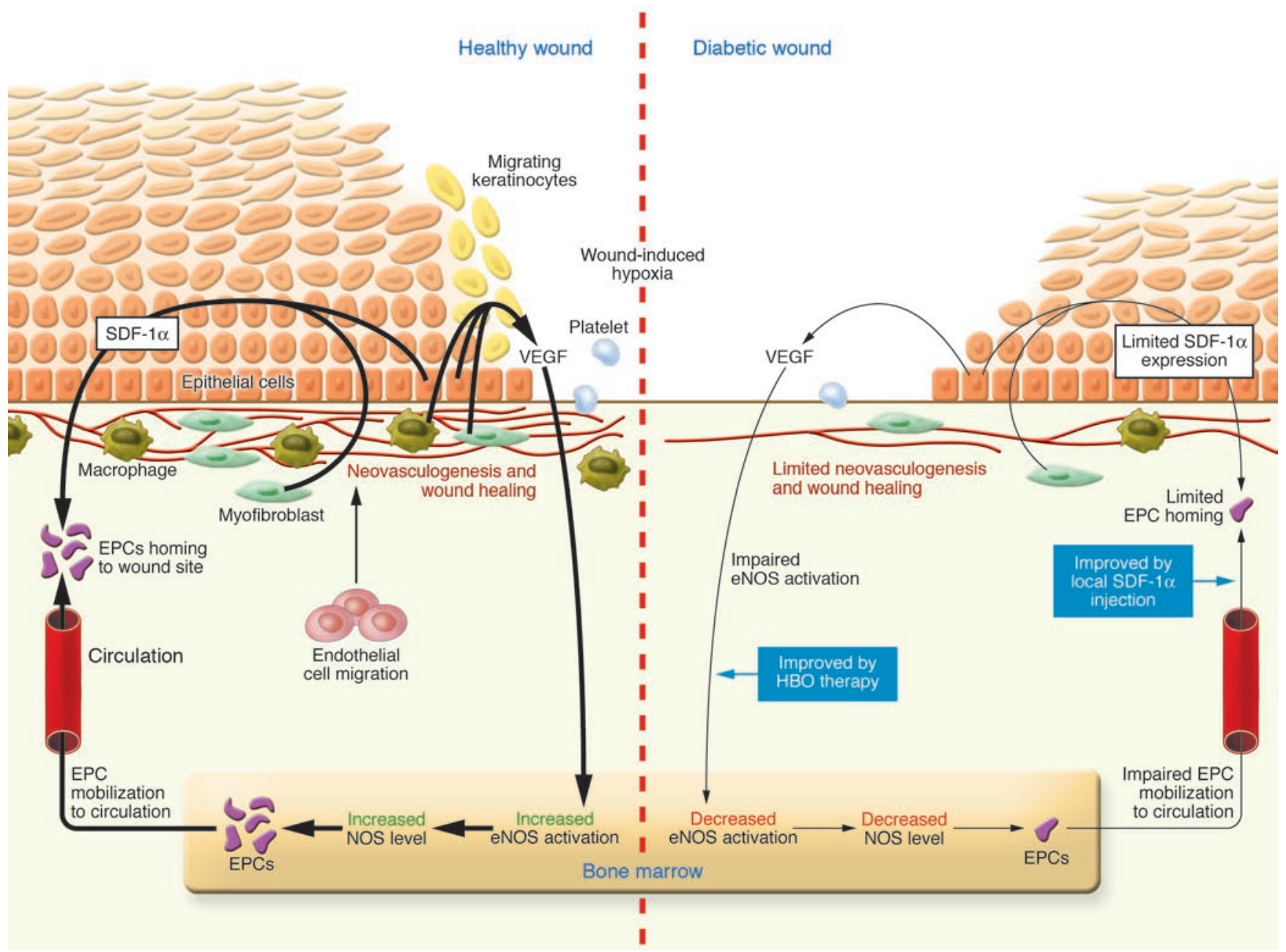

Figure 1

Mechanisms of wound healing in healthy people versus people with diabetes. In healthy individuals (left), the acute wound healing process is guided and maintained through integration of multiple signals (in the form of cytokines and chemokines) released by keratinocytes, fibroblasts, endothelial cells, macrophages, and platelets. During wound-induced hypoxia, VEGF released by macrophages, fibroblasts, and epithelial cells induces the phosphorylation and activation of eNOS in the bone marrow, resulting in an increase in NO levels, which triggers the mobilization of bone marrow EPCs to the circulation. The chemokine SDF-1 $\alpha$ promotes the homing of these EPCs to the site of injury, where they participate in neovasculogenesis. In this issue of the $\mathrm{JCl}$, Gallagher et al. (18) show that, in a murine model of diabetes (right), eNOS phosphorylation in the bone marrow is impaired, which directly limits EPC mobilization from the bone marrow into the circulation. They also show that $S D F-1 \alpha$ expression is decreased in epithelial cells and myofibroblasts in the diabetic wound, which prevents EPC homing to wounds and therefore limits wound healing. The authors further show that establishing hyperoxia in wound tissue (via HBO therapy) activated many NOS isoforms, increased NO levels, and enhanced EPC mobilization to the circulation. However, local administration of SDF-1 $\alpha$ was required to trigger homing of these cells to the wound site. These results suggest that HBO therapy combined with SDF-1 $\alpha$ administration may be a potential therapeutic option to accelerate diabetic wound healing alone or in combination with existing clinical protocols.

In contrast, cells from an adjacent nonulcerated area display the appearance of a normal phenotype but are still physiologically impaired. However, they are able to respond to administration of additional growth factors or cellular therapy. Microarray analyses of patient biopsies have confirmed these clinical findings by showing that the transcription profiles of epithelial cells from the two locations (callus and adjacent nonulcerated skin) are distinct and recognizable (14).

\section{Molecular surgery: integrative approach to healing}

Proper debridement, defined as the removal of hyperkeratotic, infected, and nonviable tissue from a wound, is essential as it accelerates wound healing $(15,16)$ via a multitude of mechanisms $(15,17)$. It is essential that pathological diagnosis is used to assure elimination of the hyperkeratotic epithelium and evaluate the extent of debridement (Figure 2). Molecular markers (13) may be utilized in the future development of rapid pathology tests for the guidance of effective debridement procedure. DFU debridement should be done in a sequential fashion until no callus or hyperkeratotic tissue is seen in the periphery of the wound and no scar or infection is present in the base of the wound.

\section{Bone marrow progenitors in diabetic wound healing}

Bone marrow-derived endothelial progenitor cells (EPCs) play a significant role in the process of neovascularization in response to 


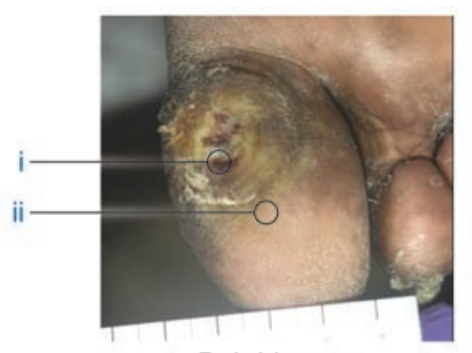

Debridement

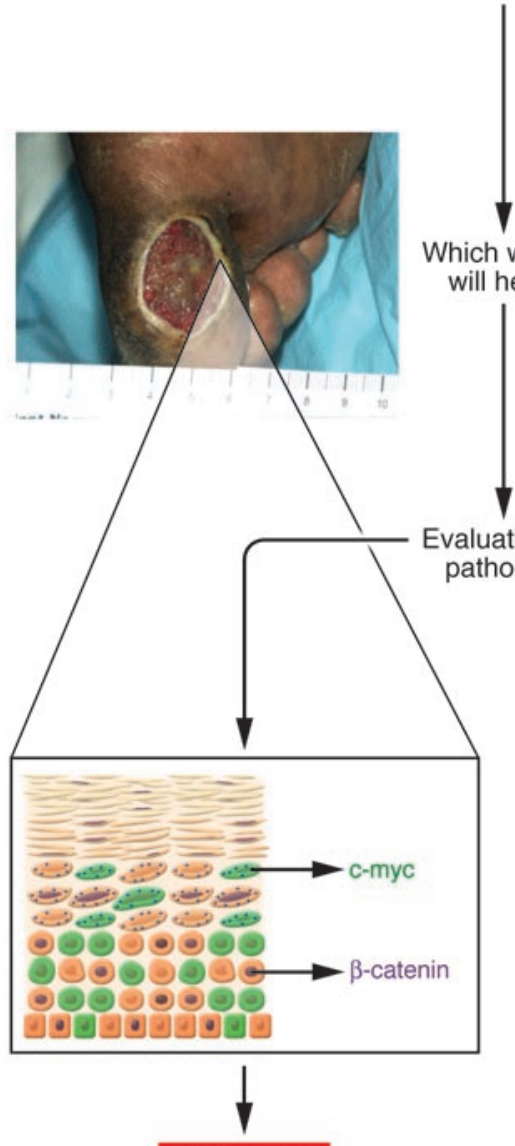

No healing
Monitor outcomes by WEMR

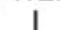

ischemic conditions, as may be the case in diabetic wounds complicated by decreased peripheral blood flow. In this issue of the JCI, Gallagher and colleagues (18) show that EPCs in the bone marrow respond to ischemia by following chemokine gradients, which results in the homing of these cells to sites of hypoxia, where they then participate in the formation of new blood vessels. Bone marrow-derived EPCs are mobilized by eNOS activation in the bone marrow, a process that the authors hypothesized is impaired in diabetics, thus preventing these cells from reaching the wound site in significant numbers. Hyperoxia has been shown to stimulate eNOS activation in some tissues (19). Therefore, to test the effect of hyperox- ia on eNOS activation and EPC recruitment, the authors wounded and later exposed diabetic and nondiabetic mice to hyperbaric oxygen therapy (HBO). Results showed that although the total numbers of active EPCs were much lower in diabetic mice than in controls, hyperoxia does indeed spur the mobilization of EPCs from the bone marrow to the bloodstream (Figure 1). EPC mobilization into the bloodstream occurs through an increase in bone marrow eNOS activation as a result of hyperoxia. The increased eNOS stimulates NO production, which in turn helps to produce EPCs from the bone marrow. Though hyperoxia can increase the levels of circulating EPCs in the bloodstream, the cells are not effectively mobilized to the

\section{Figure 2}

Molecular basis of debridement. A typical foot ulcer in a person with diabetes is shown at top. (i) The nonhealing edge (callus) containing ulcerogenic cells with molecular markers indicative of healing impairment. (ii) Phenotypically normal but physiologically impaired cells, which can be stimulated to heal. Despite a wound's appearance after debridement, it may not be healing and may need to be evaluated for the presence of molecular markers of inhibition and/or hyperkeratotic tissue (e.g., c-myc and $\beta$-catenin). We expect more such molecular markers will be identified in the future. Once a wound is debrided, pathology analyses along with immunohistochemistry should determine whether the extent of debridement was sufficient. If the extent of debridement was not sufficient (lower left diagram), cells positive for c-myc (green) and nuclear $\beta$-catenin (purple) will be found, indicating the presence of ulcerogenic cells, which will prevent the wound from healing and indicate that additional debridement is necessary. Lack of healing is also demarcated by a thicker epidermis, thicker cornified layer, and presence of nuclei in the cornified layer. If the debridement was successful (lower right lower diagram), no staining for c-myc or $\beta$-catenin will be found, indicating an absence of ulcerogenic cells and successful debridement. These markers of inhibition are useful, but the most important goal is actual healing as defined by the appearance of new epithelium, decreased area of the wound, and no drainage. This information should be stored electronically in the Wound Electronic Medical Record (WEMR), which provides an objective analysis coupled with pathology and microbiology reports. 
that the decreased expression of $S D F-1 \alpha$ by epithelial cells and myofibroblasts may be responsible for the lack of EPC homing to the periphery of diabetic wounds. This work is extremely important because it underscores the complexity of regulatory responses in diabetic wounds and explains the inconsistent response to currently approved hyperoxia protocols (i.e., HBO) in patients with diabetes (20).

\section{From bench to bedside}

A combination of therapeutic approaches is likely to lead to a successful treatment outcome for diabetic wounds. Although EPCs seem to be ideal candidates for in vivo cellbased therapies for ischemia, Gallagher and colleagues show here that singularly targeting one aspect of EPC function is not completely efficient in correcting diabetic vascular complications due to the deficits affecting both the cellular and cytokine-mediated responses to ischemia (18). Therefore, future therapies for ischemic complications will have to be based on correcting multiple deficits simultaneously. Therapeutic interventions, including correcting both EPC activation via $\mathrm{HBO}$ and EPC homing via administration of SDF- $1 \alpha$, may significantly accelerate diabetic wound healing by correcting the EPC deficit inherent to diabetic wounds.

Clinically, approved evidence-based protocols based on adequate off-loading (21) coupled with the use of FDA-approved biological therapies that have undergone rigorous controlled randomized trials $(5,22)$ must be utilized. These FDA-approved treatments are clinically efficacious and include PDGF-BB (23), fibroblasts delivered in an absorbable mesh (24), and fibroblasts and keratinocytes delivered in type 1 collagen (25). If simultaneously combined with current therapies, potential treatments targeting eNOS activation and EPC recruitment might further stimulate healing. Wound progression should be monitored with a Wound Electronic Medical Record. This allows nonhealing wounds (those that have not healed after 2 weeks of treatment) to be objectively measured and treatment to be tailored accordingly.

From the laboratory perspective, there is a compelling need to develop technologies to rapidly convert current discoveries into effective therapy for people with diabetes as well as to provide understanding of the molecular and cellular etiologies of patients with foot ulcers. Identification of the DFU genome and correlation of transcriptional profiles with clinical outcomes will determine specific genes that prevent a wound from healing and should further be utilized to develop therapies to prevent wound progression and promote rapid healing.

\section{The promise of the future}

Technologies for various molecular analyses (such as genomics, proteomics, transgenic mice), systems for sustained topical delivery (such as polymers and adenovirus vectors), major advances in tissue engineering (such as human skin engineering, cellular matrices, and bone marrow-derived cell therapy), novel discoveries of disease molecular pathogenesis from studies of patient biopsies and animal models, and developments in molecular targeting (in areas such as antisense oligonucleotides, siRNA, antibodies, and small molecules), coupled with breakthroughs in stem cell research, hold the promise of a bright future. All of these powerful technologies could potentially be applied to people with diabetic wounds in the near future. One of the major remaining steps is the integration of these resources into a coordinated effort to make the technology developed at the bench available to patients at the bedside. It is these synergistic therapies, starting at the molecular and cellular level, that will help to eliminate amputations in patients with diabetes.

\section{Acknowledgments}

We are very grateful to members of the Brem and Tomic laboratories for their assistance and helpful comments, especially Lisa Martínez and Irena Pastar. We apologize to our colleagues whose contributions were not cited due to space limitations. Our research is supported by the NIH grants R01NR08029, R01LM008443, and DK0859424.

Address correspondence to: Harold Brem, Department of Surgery, Columbia University, 5141 Broadway, Rm. 3020, New York, New York 10034, USA. Phone: (212) 932-4325; Fax: (212) 658-9806; E-mail: hb2133@ columbia.edu.

1. National Diabetes Information Clearinghouse. National Diabetes Statistics fact sheet. http://diabetes. niddk.nih.gov/dm/pubs/statistics/index.htm.

2. Wild, S., Roglic, G., Green, A., Sicree, R., and King, H. 2004. Global prevalence of diabetes: estimates for the year 2000 and projections for 2030. Diabetes Care. 27:1047-1053.

3. Reiber, G.E., et al. 1999. Causal pathways for incident lower-extremity ulcers in patients with diabetes from two settings. Diabetes Care. 22:157-162.

4. Reiber, G.E., Boyko, E.J., and Smith, D.G. 1995. Lower extremity foot ulcers and amputations in diabetes. In Diabetes in America. M.I. Harris and M.P. Stern, editors. U.S. Government Printing Office. Bethesda, Maryland, USA. 409-428.

5. Brem, H., Sheehan, P., Rosenberg, H.J., Schneider, J.S., and Boulton, A.J. 2006. Evidence-based protocol for diabetic foot ulcers. Plast. Reconstr. Surg. 117:193S-209S; discussion 210S-211S.

6. Galkowska, H., Wojewodzka, U., and Olszewski, W.L. 2006. Chemokines, cytokines, and growth factors in keratinocytes and dermal endothelial cells in the margin of chronic diabetic foot ulcers. Wound Repair Regen. 14:558-565.

7. Goren, I., Muller, E., Pfeilschifter, J., and Frank, S. 2006. Severely impaired insulin signaling in chronic wounds of diabetic ob/ob mice: a potential role of tumor necrosis factor-alpha. Am. J. Pathol. 168:765-777.

8. Falanga, V. 2005. Wound healing and its impairment in the diabetic foot. Lancet. 366:1736-1743.

9. Galiano, R.D., et al. 2004. Topical vascular endothelial growth factor accelerates diabetic wound healing through increased angiogenesis and by mobilizing and recruiting bone marrow-derived cells. Am. J. Pathol. 164:1935-1947.

10. Maruyama, K., et al. 2007. Decreased macrophage number and activation lead to reduced lymphatic vessel formation and contribute to impaired diabetic would healing. Am. J. Pathol. 170:1178-1191.

11. Gibran, N.S., et al. 2002. Diminished neuropeptide levels contribute to the impaired cutaneous healing response associated with diabetes mellitus. J. Surg. Res. 108:122-128.

12. Lobmann, R., et al. 2002. Expression of matrixmetalloproteinases and their inhibitors in the wounds of diabetic and non-diabetic patients. Diabetologia. 45:1011-1016.

13. Stojadinovic, O., et al. 2005. Molecular pathogenesis of chronic wounds: the role of beta-catenin and c-myc in the inhibition of epithelialization and wound healing. Am. J. Pathol. 167:59-69.

14. Brem, H., et al. 2007. Molecular markers in patients with chronic wounds to guide surgical debridement. Mol. Med. In press.

15. Saap, L.J., and Falanga, V. 2002. Debridement performance index and its correlation with complete closure of diabetic foot ulcers. Wound Repair Regen. 10:354-359.

16. Steed, D.L., Donohoe, D., Webster, M.W., and Lindsley, L. 1996. Effect of extensive debridement and treatment on the healing of diabetic foot ulcers. Diabetic Ulcer Study Group. J. Am. Coll. Surg. 183:61-64.

17. Steed, D.L. 2004. Debridement. Am. J. Surg. 187:71S-74S.

18. Gallagher, K.A., et al. 2007. Diabetic impairments in NO-mediated endothelial progenitor cell mobilization and homing are reversed by hyperoxia and SDF-1 $\alpha$. J. Clin. Invest. 117:1249-1259. doi:10.1172/ JCI29710.

19. Potter, C.F., et al. 1999. Effects of hyperoxia on nitric oxide synthase expression, nitric oxide activity, and lung injury in rat pups. Pediatr. Res. 45:8-13.

20. Kranke, P., Bennett, M., Roeckl-Wiedmann, I., and Debus, S. 2004. Hyperbaric oxygen therapy for chronic wounds. The Cochrane Library. http://www.thecochranelibrary.com.

21. Boulton, A.J., Vileikyte, L., Ragnarson-Tennvall, G., and Apelqvist, J. 2005. The global burden of diabetic foot disease. Lancet. 366:1719-1724.

22. Boulton, A.J., Kirsner, R.S., and Vileikyte, L. 2004. Clinical practice. Neuropathic diabetic foot ulcers. N. Engl. J. Med. 351:48-55.

23. Smiell, J.M. 1998. Clinical safety of becaplermin (rhPDGF-BB) gel. Becaplermin Studies Group. Am. J. Surg. 176:68S-73S.

24. Marston, W.A., Hanft, J., Norwood, P., and Pollak, R. 2003. The efficacy and safety of Dermagraft in improving the healing of chronic diabetic foot ulcers: results of a prospective randomized trial. Diabetes Care. 26:1701-1705.

25. Brem, H., Balledux, J., Bloom, T., Kerstein, M.D., and Hollier, L. 2000. Healing of diabetic foot ulcers and pressure ulcers with human skin equivalent: a new paradigm in wound healing. Arch. Surg. 135:627-634. 